\title{
Coupled fracture mode of a cracked disc under anti-plane loading
}

\author{
Les P. Pook ${ }^{1}$, Filippo Berto ${ }^{2, a}$, Alberto Campagnolo ${ }^{2}$ and Paolo Lazzarin ${ }^{2}$ \\ ${ }^{1} 21$ Woodside Road, Sevenoaks TN13 3HF, UK \\ ${ }^{2}$ Department of Management and Engineering, University of Padova, Stradella S.Nicola 3, \\ 36100 Vicenza, Italy
}

\begin{abstract}
The existence of three-dimensional effects at cracks has been known for many years, but understanding has been limited, and for some situations still is. Understanding improved when the existence of corner point singularities and their implications became known. Despite increased understanding, three-dimensional effects are sometimes ignored in situations where they may be important. The purpose of the present investigation is to study by means of accurate 3D finite element (FE) models a coupled fracture mode generated by anti-plane loading of a straight through-the-thickness crack in linear elastic discs. The results obtained from the highly accurate finite element analyses have improved understanding of the behaviour of through cracked discs under anti-plane loading. The influence of plate bending is increasingly important as disc thickness decreases. It appears that a new field parameter, probably a singularity, is needed to describe the stresses at the disc surfaces. Calculation of the strain energy density (SED) in a control volume at the crack tip shows that the position of the maximum SED is a function of disc thickness.
\end{abstract}

\section{Introduction}

Displacements are of fundamental importance in the understanding of the mechanics of materials in general. In particular, a fundamental fracture mechanics concept is that of crack surface displacement. Crack tip surface displacements in the vicinity of a corner point in which a crack front intersects a surface are often of practical interest. Assuming that Poisson's ratio, $v>0$, then kinematics considerations for an antisymmetric loading [1] show that modes II and III crack tip surface displacements cannot exist in isolation. Mode II induces mode $\mathrm{III}^{\mathrm{c}}$ and mode III induces mode $\mathrm{II}^{\mathrm{c}}$. The resulting combination of modes II and III is sometimes called the antisymmetric mode. Coupled modes have been demonstrated by the use of foam plastic models $[2,3]$.

In three dimensional geometries, the derivation of stress intensity factors makes the implicit assumption that a crack front is continuous. This is not the case in the vicinity of a corner point, and the nature of the crack tip singularity changes. The resulting corner point singularities were described in detail in 1979 by Bažant and Estenssoro [4]. Some additional results were given by Benthem in 1980 [5].

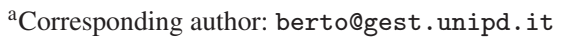

This is an Open Access article distributed under the terms of the Creative Commons Attribution License 4.0, which permits unrestricted use, distribution, and reproduction in any medium, provided the original work is properly cited. 

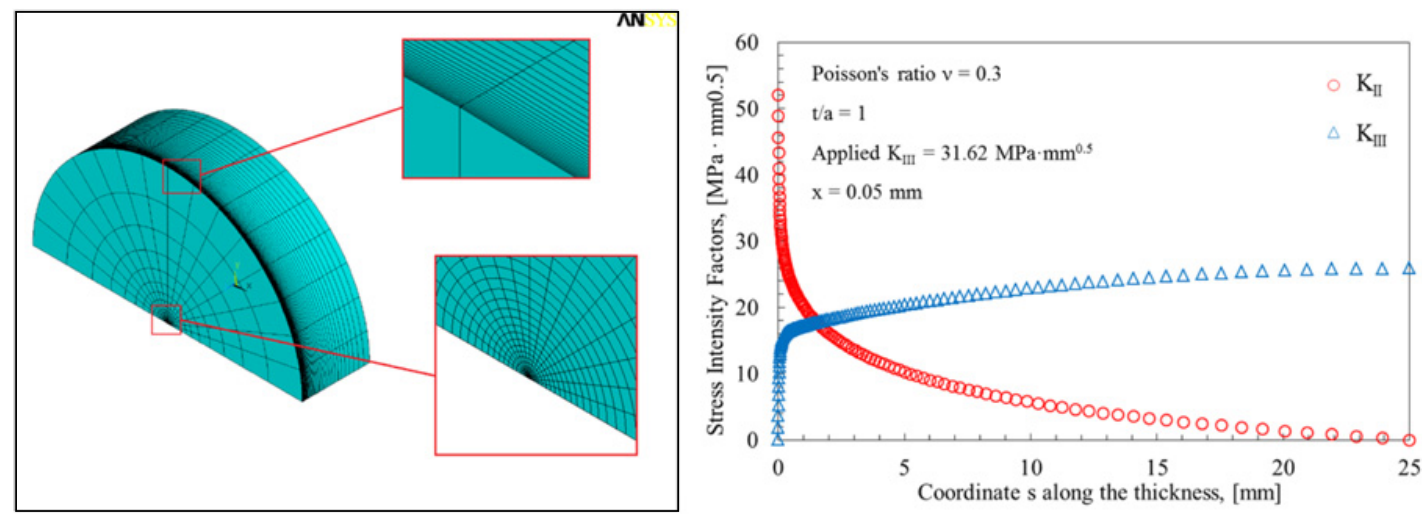

Figure 1. (a) Overall view of finite element mesh. Detail of finite element mesh at outer surface and at crack tip. (b) Through thickness distribution of $K_{\mathrm{II}}$ and $K_{\mathrm{III}}$ for $t / a=1$.

There do not appear to be any exact analytic solutions for corner point singularities. At the present state of the art the extent of a corner point singularity dominated region has to be determined numerically.

There does not appear to have been a systematic investigation of the extent to which Bažant and Estenssoro's initial assumption is justified. Their assumption does appear to be satisfactory for the symmetric mode (mode I) in that their analysis leads to useful results [2]. The purpose of the present investigation is to study by means of accurate three dimensional finite element (FE) models a coupled fracture mode generated by anti-plane loading of a straight through-the-thickness crack in linear elastic discs. The material is assumed to be a homogeneous isotropic continuum, and its behaviour is assumed to be linearly elastic.

\section{Finite element modeling}

Stresses, stress intensity factors and displacements were examined in detail for $100 \mathrm{~mm}$ diameter discs of various thicknesses under anti-plane (nominal mode III) loading. The disc radius, $r$, is $50 \mathrm{~mm}$, and the thickness is $t$. A through thickness crack has its tip at the centre of the disc, so its length, $a$, is $50 \mathrm{~mm}$. Calculations were carried out using ANSYS 11 for $\mathrm{t} / \mathrm{a}=0.25,0.5,0.75,1,1.25,1.5,1.75,2$, $2.25,2.5,2.75$ and 3 . One quarter of the disc was modelled. An overall view of the finite element mesh is shown in Fig. 1a. Poisson's ratio was taken as 0.3 and Young's modulus as $200 \mathrm{GPa}$. Displacements corresponding to $K_{\mathrm{III}}=1 \mathrm{MPa} \cdot \mathrm{m}^{0.5}\left(31.62 \mathrm{~N} \cdot \mathrm{mm}^{0.5}\right)$ were applied to the cylindrical surface. Stress intensity factors were calculated from stresses near the crack tip using standard equations $[1,3]$.

\section{Results}

Through thickness distributions of $K_{\mathrm{II}}$ and $K_{\mathrm{III}}$ for $t / a=1$ are shown in Fig. 1b. Results for other values of $t / a$ are generally similar, but with some differences in detail. Maximum value of $K_{\mathrm{III}}$ is at the centreline.

For the thicker discs $K_{\text {III }}$ is nearly constant for $s>50 \mathrm{~mm}$, and then decreases steadily towards the surface, with an abrupt drop close to the surface.

Plate bending theory [1] suggests that $K_{\text {II }}$ should be zero on the centre line, with a linear increase towards a surface. The influence of plate bending increases as $t / a$ decreases, and for $t / a=0.25$ the distribution of $K_{\mathrm{II}}$ is nearly linear with a greater increase towards the surface. Maximum values of $K_{\mathrm{II}}$ are at the surface (Fig. 1b). 


\section{Strain energy density through the disc thickness}

The intensity of the local stress and strain state through the disc thickness can be easily evaluated by using the strain energy density (SED) averaged over a control volume embracing the crack tip (see Ref. [6] for a review of the SED approach). The main advantage of the SED parameter with respect to the local stress-based parameters is that it does not need very refined meshes [7].

The local SED variation across the disc averaged over a cylindrical volume having radius $R_{0}$ and height $h$, with $h$ about equal to $R_{0}$, has been investigated. In Refs [8-10] $R_{0}$ was thought of as a material property which varies under static and fatigue loading but here, for the sake of simplicity, $R_{0}$ and $h$ are simply set equal to $1.0 \mathrm{~mm}$.

It has been observed that the position of the maximum SED changes from case to case. It is close to the lateral surface for the two cases $\mathrm{t} / \mathrm{a}=0.5$ and 1.0. For larger thicknesses the maximum value of the SED is at the mid-plane.

\section{Conclusions}

The results obtained from the highly accurate finite element analyses have improved understanding of the behaviour of through cracked discs under anti-plane loading. In particular, it is confirmed that mode III does induce coupled mode II $^{\mathrm{c}}$.

The influence of plate bending is increasingly important as disc thickness decreases.

The anti-plane loading used is a nominal mode III loading. For thin discs it is a mixed modes III and II loading, in which mode III induces mode II $^{\mathrm{c}}$ and vice versa.

It appears that a new field parameter, probably a singularity, is needed to describe the stresses at the disc surfaces.

Calculation of the strain energy density (SED) in a control volume at the crack tip shows that the position of the maximum SED is a function of disc thickness. For thin discs it is close to the lateral surface, but for thick discs the maximum SED is at the mid-plane.

\section{References}

[1] A. Kotousov, P. Lazzarin, F. Berto, L.P. Pook, Eng. Fract. Mech. 108, 65-74 (2013)

[2] L.P. Pook, Fatigue Fract. Engng. Mater. Struct. 36, 699-723 (2013)

[3] L.P. Pook, Fatigue Fract. Engng. Mater. Struct. 23, 141-150 (2000)

[4] Z.P. Bažant, L.F. Estenssoro, Int. J. Solids Struct. 15, 405-426 (1979)

[5] J.P. Benthem, Int. J. Solids Struct. 16(2), 119-130 (1980)

[6] F. Berto, P. Lazzarin, Mater. Sci. Eng. R 75, 1-48 (2014)

[7] P. Lazzarin, F. Berto, M. Zappalorto, Int. J. Fatigue 32, 1559-1567 (2010)

[8] P. Lazzarin, F. Berto, M. Elices, J. Gómez, Fatigue Fract. Engng, Mater. Struct, 32, 671-684 (2009)

[9] F. Berto, P. Lazzarin, J.R. Yates, Fatigue Fract. Engng. Mater. Struct, 34, 921-943 (2011)

[10] P. Lazzarin, F. Berto, B. Atzori, Theor. Appl. Fract. Mech. 63-64, 32-39 (2013) 